\title{
ANÁlISIS DE LA DISTRIBUCIÓN Y DENSIDAD ESTOMÁTICA EN TRICHLORIS (Chloridoideae, PoAceae), Bajo difERENTES Regímenes hídricos
}

\author{
MARÍA A. TROD ${ }^{1,2, *}$, PABLO A. TOMAS ${ }^{1}$, GERARDO D. L. CERVIGNI², JUAN M. ZABALA ${ }^{1}$, \\ LORENA D. R. MARINONI ${ }^{1,2}$ y JULIO A. GIAVEDONI ${ }^{1}$
}

\begin{abstract}
Summary: Analisys of distribution and stomatal density in Trichloris (Chloridoideae, Poaceae), under different water regimes. Trichloris crinita and T. pluriflora are perennial grasses native from Argentina that have productive potential as forage for arid and semi-arid regions. The objectives of the present work were to analyze in both species the variations in leaf stomatal distribution and density under two different water regimes and their association with rainfall at the place of origin. Six introductions for each species were characterized. Two trials were implemented, one in a humid environment and the other in a dry environment. Measurements of foliar dimensions and stomatal counts in each material for each environment were made. The Principal Component Analysis represented $73.3 \%$ of the variation on the first two axes, responding to the effect of the evaluation environment on stomatal parameters and to the differences between species for their foliar dimensions. Significant differences were observed between introductions of $T$. crinita in both environments for most of the variables analyzed. Variations between introductions of $T$. pluriflora were observed only in humid environment. The observed variations correlated with rainfall at the collection site only for $T$. crinita. The variables used here were useful for the characterization of the materials analyzed; their implications for the conservation of this plant genetic resource are discussed.
\end{abstract}

Key words: Genetics resources, grasses, Leptochloa, Trichloris crinita, Trichloris pluriflora, stomatal density.

\begin{abstract}
Resumen: Trichloris crinita y T. pluriflora son gramíneas perennes nativas de Argentina que presentan potencialidad productiva como forrajeras para regiones áridas y semiáridas. Los objetivos del presente trabajo fueron analizar para ambas especies las variaciones en la distribución y densidad estomática foliar ante dos regímenes hídricos diferentes y su asociación con las precipitaciones en el lugar de origen. Se caracterizaron seis introducciones para cada especie, implantando dos ensayos, uno en ambiente húmedo y otro en ambiente seco, realizándose mediciones de dimensiones foliares y recuentos estomáticos en cada material para cada ambiente. El Análisis de Componentes Principales representó el 73,3\% de la variación sobre los dos primeros ejes, respondiendo al efecto del ambiente de evaluación sobre los parámetros estomáticos y a las diferencias entre especies para las dimensiones foliares. Para $T$. crinita se observaron diferencias significativas entre introducciones para la mayoría de las variables analizadas en ambos ambientes. Para T. pluriflora, las variaciones entre las introducciones fueron evidentes solamente en el ambiente húmedo. Las variaciones observadas presentaron correlación con las precipitaciones en el sitio de colecta únicamente para T. crinita. Las variables aquí empleadas resultaron de utilidad para la caracterización de los materiales analizados; se discute su implicancia para la conservación del recurso fitogenético.
\end{abstract}

Palabras clave: Densidad estomática, gramíneas, Leptochloa, recursos genéticos, Trichloris crinita, Trichloris pluriflora.

\footnotetext{
${ }^{1}$ Cátedra de Genética y Mejoramiento Vegetal, Facultad de Ciencias Agrarias, Universidad Nacional del Litoral, RP Luis Kreder 2805, 3080 HOF Esperanza, Argentina.

${ }^{2}$ Consejo Nacional de Investigaciones Científicas y Técnicas, Buenos Aires, Argentina.

*maria_trod@hotmail.com
} 


\section{INTRODUCCIÓN}

En las últimas décadas, el avance de las fronteras productivas hacia áreas no cultivadas ha sido una de las principales causas de la erosión genética de los pastizales naturales (FAO, 2008; Henwood, 2010; Carbutt et al., 2017). Frente a este escenario, la conservación de la variabilidad de genotipos locales adaptados a diversos ambientes para su aprovechamiento productivo constituye uno de los pilares de la valorización de los recursos fitogenéticos a nivel mundial.

Entre las especies nativas de Argentina con mayor potencial productivo para regiones áridas y semiáridas con clima subtropical se encuentran Trichloris crinita (Lag.) Parodi y T. pluriflora E. Fourn. (Chloridoideae, Poaceae). Mientras que a $T$. crinita se la encuentra desde las provincias de Neuquén y Río Negro hasta el Norte del país, abarcando las provincias fitogeográficas del Monte y Chaqueña (Zuloaga et al., 1994), T. pluriflora presenta una distribución restringida al Chaco Húmedo (Marinoni et al., 2015). Ambas especies son $\mathrm{C} 4$, perennes, de reproducción sexual y autógama (Gutierrez et al., 2016; Kozub et al., 2017) y han sido estudiadas como potencial recurso forrajero debido a la diversidad de ambientes marginales donde se las encuentra y a su tolerancia a factores abióticos como sequía y salinidad (Passera et al., 1997; Greco \& Cavagnaro, 2003). Si bien recientemente estas especies han sido incluidas dentro del género Leptochloa como Leptochloa crinita (Lag.) P.M. Peterson \& N.W. Snow y Leptochloa pluriflora (E. Fourn.) P.M. Peterson \& N.W. Snow (aceptados por Flora Argentina, http:// www.floraargentina.edu.ar) en base a un estudio de filogenia molecular (Peterson et al., 2015), en el presente trabajo se mantiene el género Trichloris, por tratarse de una caracterización a nivel inter e intraespecífico de las únicas dos especies de este género y porque en estudios de índole agronómica son los nombres científicos comúnmente utilizados.

Una de las consecuencias primarias del estrés hídrico en la planta es la reducción de la actividad fotosintética debido a efectos estomáticos, los cuales consisten en la reducción del intercambio gaseoso y están directamente relacionados con el potencial hídrico foliar (Kaiser, 1987; Baruch \& Fernández, 1993). Las especies tolerantes son capaces de soportar potenciales hídricos más bajos, lo que les permite sostener la actividad fotosintética y alcanzar mayores tasas de crecimiento relativo en condiciones de estrés (Ludlow \& Ng, 1976; Xu \& Zhou, 2008). Esa tolerancia a estrés hídrico en ecotipos vegetales provenientes de ambientes restrictivos es el resultado de adaptaciones fisiológicas, morfológicas y anatómicas en los individuos, las que han sido evolutivamente seleccionadas para hacer más eficiente el uso del agua (De Micco \& Aronne, 2012; Ashraf \& Harris, 2013). Dimensiones foliares reducidas y cambios en la densidad y distribución de estomas en la epidermis foliar constituyen aspectos relacionados con la adaptación a una baja disponibilidad hídrica durante el período de crecimiento (Redmann, 1985; Hong et al., 2000; Villagra et al., 2011; González Medrano, 2012). Además, esos caracteres pueden variar en diferente medida entre años en función de las condiciones ambientales, lo que confiere plasticidad adaptativa en ambientes cambiantes y áreas de transición (Sharma, 1972; Barboza et al., 2006; Fraser et al., 2009; Muir, 2015). Greco \& Cavagnaro (2003), han reportado para $T$. crinita la capacidad de resistir muy bajos potenciales hídricos en la hoja antes de verse afectado el crecimiento, aunque se desconocen los mecanismos y adaptaciones involucradas. La amplia distribución geográfica tanto de $T$. crinita como de T. pluriflora sugiere que ambas especies presentan mecanismos que le permiten adaptarse a las diferentes condiciones hídricas de los ambientes donde vegetan. Asimismo, la diversidad y plasticidad para parámetros foliares y estomáticos propios de cada procedencia podría tener relación con las diferentes condiciones hídricas de los sitios de origen.

Los objetivos del presente trabajo fueron analizar la distribución y densidad estomática en láminas foliares, sus variaciones ante diferentes condiciones ambientales y su asociación con las precipitaciones en el lugar de origen para materiales de Trichloris procedentes de ambientes contrastantes.

\section{Materiales y Métodos}

\section{Material vegetal}

Se caracterizaron seis introducciones de $T$. crinita y seis de $T$. pluriflora procedentes de diferentes regiones de Argentina, facilitadas por el Banco de Germoplasma del Programa de Documentación, Conservación y Valoración de la Flora Nativa 


\section{A. Trod et al. - Distribución y densidad estomática en Trichloris}

(ProDoCoVa) de la Facultad de Ciencias Agrarias, Universidad Nacional del Litoral (Tabla 1). Las semillas se pusieron a germinar en macetas con tierra $\mathrm{y}$ arena, en partes iguales y posteriormente llevadas a campo en noviembre de 2013. Los ensayos se implantaron en dos ambientes, denominados Esperanza (ambiente húmedo) y Catamarca (ambiente seco), localizado el primero en el campo experimental de la Facultad de Ciencias Agrarias, UNL (Esperanza, Santa Fe; 31 27’02” S, 6055'56" O) y el segundo en la Estación Experimental

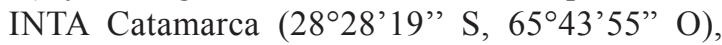
representando ambientes contrastantes en cuanto a registros de precipitaciones y temperaturas durante la temporada de crecimiento (Fig. 1). En ambos sitios los genotipos se ubicaron en un diseño completamente aleatorizado con 5 repeticiones por introducción, considerando a cada individuo como una repetición. Para evitar el efecto del trasplante, las determinaciones se realizaron en la segunda temporada de crecimiento a campo.

\section{Determinación de variables}

En el mes de febrero de 2015, al inicio de la etapa reproductiva, se tomó como muestra la lámina foliar completamente expandida e inmediata inferior a la hoja bandera de cinco macollos por genotipo. Las muestras fueron fijadas y preservadas en FAA 18:1:1 (etanol: ác. acético: formol) hasta su análisis.

Mediante medición directa se determinó el Ancho de Lámina (AL) en milímetros para la región central de la hoja muestreada. Los recuentos estomáticos se realizaron sobre secciones de láminas foliares tratadas mediante la técnica de Metcalfe modificada (Zarlavsky, 2014), utilizando un microscopio óptico Olympus CH30 de campo claro, objetivo de 40X y ocular graduado. Se realizaron recuentos en al menos 10 campos al azar para cada muestra en la región central de la lámina y se determinaron las siguientes variables:

- Estomas por milímetro lineal de región intercostal en la epidermis abaxial (ELAB)

- Estomas por milímetro lineal de región intercostal en la epidermis adaxial (ELAD)

- Estomas por $\mathrm{mm}^{2}$ en la epidermis abaxial (ESAB)

- Estomas por $\mathrm{mm}^{2}$ en la epidermis adaxial (ESAD)

- Porcentaje de estomas $\mathrm{mm}^{2}$ en la epidermis adaxial/estomas $\mathrm{mm}^{2}$ de lámina $(\% \mathrm{AD})$.

Considerando que los estomas en Trichloris $s p$. se ubican en forma lineal en la región intercostal

Tabla 1. Detalle de las introducciones incluidas en este estudio y parámetros geográficos y ambientales de su respectivo lugar de origen, proporcionados por ProDoCoVa.

\begin{tabular}{|c|c|c|c|c|c|c|}
\hline \multirow{2}{*}{ Especie } & \multirow{2}{*}{ Introducción } & \multirow{2}{*}{ Provincia } & \multicolumn{2}{|c|}{ Coordenadas geográficas } & \multirow{2}{*}{$\begin{array}{l}\text { Altitud } \\
\text { (msnm) }\end{array}$} & \multirow{2}{*}{$\begin{array}{c}\text { Precipitaciones } \\
\text { (mm anuales) }\end{array}$} \\
\hline & & & Latitud (S) & Longitud (0) & & \\
\hline \multirow{6}{*}{ Trichloris crinita } & 7320 & Mendoza & $-34,7721$ & $-68,6117$ & 1209 & 314 \\
\hline & 7390 & San Juan & $-31,7254$ & $-67,8462$ & 639 & 180 \\
\hline & 7602 & Córdoba & $-29,8500$ & $-64,6697$ & 191 & 516 \\
\hline & 8492 & Formosa & $-25,1180$ & $-59,9786$ & 116 & 930 \\
\hline & 8608 & Santa Fe & $-28,7011$ & $-60,0799$ & 62 & 1142 \\
\hline & 9550 & San Luis & $-26,8684$ & $-66,3150$ & 399 & 446 \\
\hline \multirow{6}{*}{ Trichloris pluriflora } & 7000 & Salta & $-25,2169$ & $-64,9295$ & 681 & 648 \\
\hline & 7491 & Catamarca & $-28,6953$ & $-65,8365$ & 412 & 390 \\
\hline & 7958 & San Luis & $-32,3616$ & $-66,1140$ & 604 & 505 \\
\hline & 7967 & San Luis & $-32,1974$ & $-65,6832$ & 478 & 542 \\
\hline & 8419 & $\begin{array}{l}\text { Sgo, del } \\
\text { Estero }\end{array}$ & $-28,5544$ & $-61,8046$ & 82 & 851 \\
\hline & 8466 & Chaco & $-26,5842$ & $-60,6222$ & 103 & 975 \\
\hline
\end{tabular}




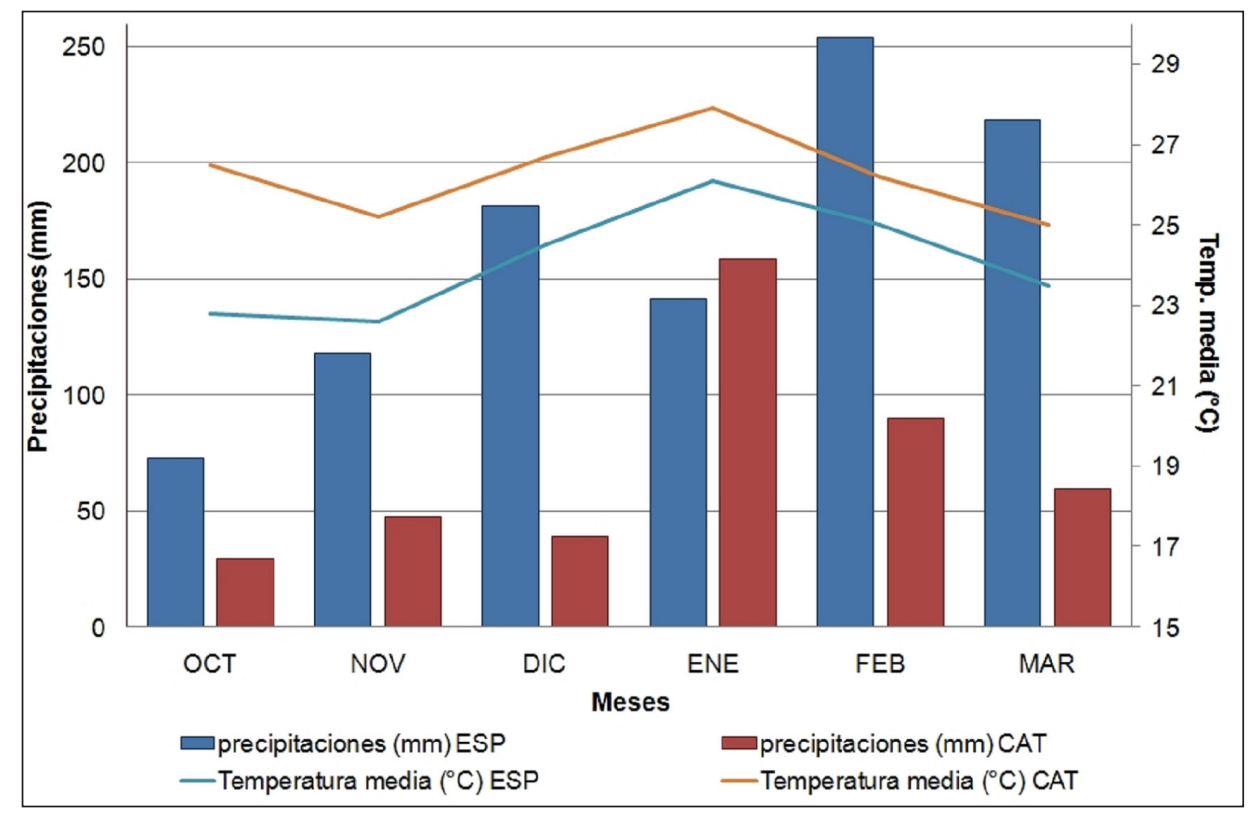

Fig. 1. Registros de temperatura media diaria y precipitaciones mensuales durante el período de evaluación (Octubre 2014 - Marzo 2015) en los dos ambientes de emplazamiento de los ensayos (ESP: Esperanza; CAT: Catamarca).

de ambas epidermis foliares (Gil et al., 1992), se registró además el número de regiones intercostales en la sección transversal de la lámina analizada, para luego estimar la densidad de esas regiones intercostales en el ancho de lámina (DRI, $\mathrm{mm}^{-1}$ ), estimada como el cociente entre el número de regiones y el ancho de lámina.

\section{Análisis estadístico}

Con el conjunto de variables analizadas se confeccionó una matriz de datos y se realizó un Análisis de Componentes Principales (ACP) para determinar la existencia de posibles patrones de variación y relaciones entre y dentro de las especies frente a las condiciones de evaluación. Para la representación gráfica se estimó el Coeficiente de Correlación Cofenético (CCC), a modo de inferir el grado de distorsión producto del análisis a partir de la matriz original de datos.

Se realizó un análisis estadístico utilizando el test t-Student para muestras apareadas $(\mathrm{p}<0,05)$, para la comparación de promedios a tres niveles de análisis: entre especies en general, entre especies dentro de cada ambiente y entre ambientes dentro de cada especie.

Posteriormente, para cada especie por separado se analizaron estadísticamente las diferencias entre introducciones dentro de cada ambiente mediante análisis de la varianza (ANVA), previo análisis de normalidad y homogeneidad de varianzas, y las medias fueron comparadas mediante el test de Tukey $(\mathrm{p}<0,05)$. Para aquellas variables que no presentaron distribución normal, se realizó un análisis estadístico no paramétrico mediante Kruskal-Wallis $(\mathrm{p}<0,05)$.

Finalmente, se determinó la correlación entre los valores determinados para las variables analizadas y las precipitaciones promedio anuales en los sitios de origen respectivos mediante el coeficiente Pearson, analizando por especie tanto en general como dentro de cada ambiente de evaluación. Todos los análisis se realizaron utilizando el software estadístico Infostat 2018 (Di Rienzo et al., 2018).

\section{Resultados}

En el análisis de componentes principales los dos primeros ejes explicaron el $73,7 \%$ de la variabilidad registrada para ambas especies de Trichloris. Sobre la CP1 (58,1\% de la variación) se observó una dispersión relacionada con el ambiente 


\section{A. Trod et al. - Distribución y densidad estomática en Trichloris}

de evaluación (Fig. 2). Los registros obtenidos en Catamarca se ubicaron sobre los cuadrantes de la izquierda y los registros obtenidos en Esperanza sobre los cuadrantes de la derecha, resultando de mayor relevancia las variables de densidad estomática en función de sus autovectores sobre esta componente (Tabla 2). Por otro lado, sobre la CP2 (15,6\% de la variación) la diferenciación fue mayormente entre especies, ubicándose T. pluriflora en los cuadrantes superiores y $T$. crinita en los cuadrantes inferiores, principalmente en función del peso de AL y DRI sobre dicha componente. En la representación gráfica, la introducción 7602 en Catamarca se localizó muy distante del resto de los materiales analizados, constituyendo el principal elemento que define la proporción de la variación que es explicada por la CP1 (Fig. 2).

Tanto los resultados de los análisis estadísticos entre especies a nivel general, como los obtenidos entre especies por ambiente y por especie entre ambientes, coincidieron con los resultados presentados en el biplot del ACP. Para el conjunto de datos, las diferencias significativas entre $T$. crinita y $T$. pluriflora se registraron para las variables AL, DRI y ELAB (Tabla 3). En general, las varianzas por especie para el conjunto de datos y dentro de cada ambiente, fueron mayores para $T$. crinita, en coincidencia con la dispersión observada para esta especie en el ACP.

Al considerar por ambiente, para Catamarca se observaron diferencias significativas entre especies solamente para las variables AL y DRI. En Esperanza, además de esas dos variables, se observaron diferencias significativas entre especies para ELAB y ELAD. Al particionar por especie se observó una situación contrapuesta a la partición por ambiente, dado que la mayoría de las variables analizadas presentaron para ambas especies diferencias significativas entre ambientes, con excepción de AL y ELAB (Tabla 3).

Analizando cada especie por separado, resultó significativa la interacción "Introducción x Ambiente", por loque el análisis de varianza serealizó para cada especie particionando por ambiente. Para T. crinita en Catamarca se observaron diferencias estadísticas significativas entre introducciones para todas las variables analizadas. Las introducciones 8492 y 8608 registraron los mayores valores para todos los parámetros, mientras que la introducción 7602 presentó los menores registros (Tabla 4). Para esta misma especie en Esperanza, se observaron diferencias significativas entre introducciones para la mayoría de las variables analizadas, con excepción de ELAB y \%AD. Al igual que en

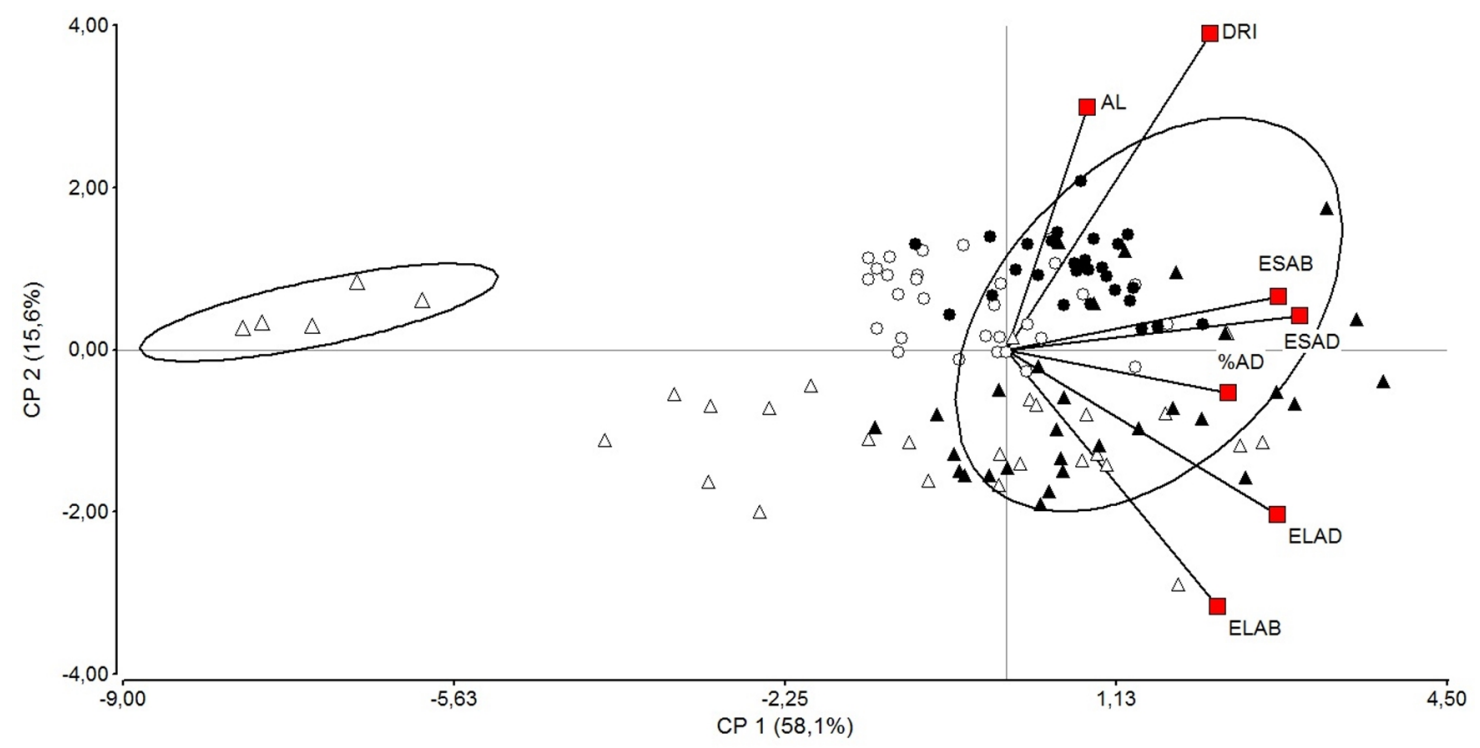

Fig. 2. Biplot del Análisis de Componentes Principales para los dos primeros ejes. $\Delta:$ T. crinita en Catamarca. $\boldsymbol{\Delta}$ : T. crinita en Esperanza. О: T. pluriflora en Catamarca. ๑: T. pluriflora en Esperanza. Las elipses de confianza (95\%) corresponden a la introducción 7602 en Catamarca (izq.) y Esperanza (der.). CCC: 0,945. 
Tabla 2. Autovectores de las variables analizadas para las dos primeras componentes principales. Valores destacados indican los coeficientes de mayor peso en cada componente.

\begin{tabular}{|ccc|}
\hline \multicolumn{3}{c}{ Autovectores } \\
\hline Variables & CP1 & CP2 \\
\hline AL & 0,13 & $\mathbf{0 , 4 8}$ \\
ELAB & 0,34 & $-\mathbf{0 , 5 1}$ \\
ELAD & $\mathbf{0 , 4 4}$ & $-0,33$ \\
ESAB & $\mathbf{0 , 4 4}$ & 0,1 \\
ESAD & $\mathbf{0 , 4 8}$ & 0,007 \\
DRI & 0,33 & $\mathbf{0 , 6 2}$ \\
\%AD & $\mathbf{0 , 3 6}$ & $-0,09$ \\
\hline
\end{tabular}

Catamarca, las introducciones 8492 y 8608 se ubicaron entre los mayores registros. Para la introducción 7602 observó un comportamiento particular: si bien presentó los menores valores de densidad estomática lineal en la superficie adaxial, registró el mayor valor de DRI, lo que incrementó significativamente su densidad estomática por unidad de superficie (ESAD y ESAB), al tiempo que, a diferencia del resto de los materiales analizados, presentó menores valores de AL que en Catamarca (Tabla 4).

Para T. pluriflora en Catamarca, la única variable que presentó diferencias significativas fue $\% \mathrm{AD}$, registrando los menores valores la introducción 7000 y los mayores valores la 7491 (Tabla 5). En Esperanza, las variables que presentaron diferencias estadísticas significativas entre materiales

Tabla 3. Valores de medias, varianzas y análisis estadístico con nivel de significancia entre especies a nivel general, entre especies particionando por ambiente y entre ambientes particionando por especie.

\begin{tabular}{|c|c|c|c|c|c|c|}
\hline \multirow{2}{*}{ Variable } & \multirow{2}{*}{ Ambiente/Especie } & \multicolumn{2}{|c|}{ T. crinita } & \multicolumn{2}{|c|}{ T. pluriflora } & \multirow{2}{*}{ p-valor } \\
\hline & & Media & Varianza & Media & Varianza & \\
\hline \multirow{4}{*}{$A L$} & General & 5 & 3,3 & 10,2 & 2,7 & $<0,0001$ \\
\hline & Catamarca & 4,8 & 2 & 10,1 & 3,6 & $<0,0001$ \\
\hline & Esperanza & 5,2 & 4,6 & 10,3 & 1,9 & $<0,0001$ \\
\hline & p-valor & 0,398 & & 0,6403 & & \\
\hline \multirow{4}{*}{ DRI } & General & 2,8 & 0,5 & 3,3 & 0,1 & 0,0001 \\
\hline & Catamarca & 2,5 & 0,3 & 3,1 & 0,1 & $<0,0001$ \\
\hline & Esperanza & 3,2 & 0,6 & 3,5 & 0,1 & 0,0354 \\
\hline & p-valor & 0,0003 & & $<0,0001$ & & \\
\hline \multirow{4}{*}{ ELAD } & General & 31,3 & 105,4 & 31,1 & 13,1 & 0,8921 \\
\hline & Catamarca & 27,3 & 162,4 & 30,1 & 14,3 & 0,2544 \\
\hline & Esperanza & 35,3 & 18,6 & 32,2 & 9,9 & 0,0022 \\
\hline & p-valor & 0,0023 & & 0,026 & & \\
\hline \multirow{4}{*}{ ELAB } & General & 42 & 93,3 & 38,2 & 23,2 & 0,0077 \\
\hline & Catamarca & 39,9 & 123,1 & 37,8 & 27,6 & 0,3495 \\
\hline & Esperanza & 44,1 & 57,5 & 38,6 & 19,2 & 0,0013 \\
\hline & p-valor & 0,0918 & & 0,5047 & & \\
\hline \multirow{4}{*}{ ESAD } & General & 183,9 & 5934,9 & 186,9 & 1077,2 & 0,7839 \\
\hline & Catamarca & 145,1 & 6392,4 & 166,9 & 841,5 & 0,169 \\
\hline & Esperanza & 222,8 & 2562,5 & 207,6 & 485,6 & 0,1421 \\
\hline & p-valor & $<0,0001$ & & $<0,0001$ & & \\
\hline \multirow{4}{*}{ ESAB } & General & 243,2 & 7810 & 250 & 1405,5 & 0,5845 \\
\hline & Catamarca & 207,6 & 7858,5 & 231,3 & 1114,8 & 0,1797 \\
\hline & Esperanza & 278,8 & 5406,7 & 269,46 & 988 & 0,5256 \\
\hline & p-valor & 0,0013 & & $<0,0001$ & & \\
\hline \multirow{4}{*}{$\% A D$} & General & 41,1 & 83,6 & 42,7 & 5,9 & 0,2111 \\
\hline & Catamarca & 37,6 & 131 & 41,8 & 4,8 & 0,054 \\
\hline & Esperanza & 44,7 & 12,8 & 43,5 & 5,7 & 0,1527 \\
\hline & $p$-valor & 0,0025 & & 0,0053 & & \\
\hline
\end{tabular}




\section{A. Trod et al. - Distribución y densidad estomática en Trichloris}

\begin{tabular}{|c|c|c|c|c|c|c|c|c|}
\hline Ambiente & Accesión & $\begin{array}{c}\mathrm{AL} \\
(\mathrm{mm})\end{array}$ & $\begin{array}{c}\text { DRI } \\
\left(\mathrm{mm}^{-1}\right)\end{array}$ & $\begin{array}{c}\text { ELAD* } \\
\text { (estomas/mm) }\end{array}$ & $\begin{array}{c}\text { ELAB* } \\
\text { (estomas/mm) }\end{array}$ & $\begin{array}{c}\text { ESAD* } \\
\text { (estomas/mm²) }\end{array}$ & $\begin{array}{c}\text { ESAB } \\
\text { (estomas } / \mathrm{mm} \text { ) }\end{array}$ & $\% \mathrm{AD}^{*}$ \\
\hline \multirow{6}{*}{ Catamarca } & 7320 & $3,6 \mathrm{~b}$ & $2,4 \mathrm{bc}$ & $31,4 a$ & 34,8 bc & $154,0 \mathrm{a}$ & $166,2 \mathrm{~cd}$ & $46,2 \mathrm{a}$ \\
\hline & 7390 & $4,8 a b$ & $2,6 \mathrm{ac}$ & $30,9 a$ & $39,8 a b$ & $166,3 \mathrm{a}$ & $212,8 \mathrm{bc}$ & $43,2 a$ \\
\hline & 7602 & $3,2 \mathrm{~b}$ & $2,0 \mathrm{c}$ & $3,8 \mathrm{~b}$ & $22,4 \mathrm{c}$ & $15,7 \mathrm{~b}$ & $88,8 \mathrm{~d}$ & $14,4 \mathrm{~b}$ \\
\hline & 8492 & $6,2 \mathrm{a}$ & $3,2 \mathrm{a}$ & $33,4 a$ & $49,3 \mathrm{a}$ & $213,8 a$ & $316,5 a$ & 40,4 a \\
\hline & 8608 & $6,0 a b$ & $2,8 a b$ & $33,6 \mathrm{a}$ & $48,8 \mathrm{a}$ & 190,5 a & $274,5 a b$ & 40,6 a \\
\hline & 9550 & $5,0 \mathrm{ab}$ & $2,1 \mathrm{bc}$ & $30,6 \mathrm{a}$ & $44,2 a b$ & $130,3 a b$ & $186,9 \mathrm{bd}$ & 40,6 a \\
\hline \multirow{6}{*}{ Esperanza } & 7320 & $4,0 \mathrm{~cd}$ & $2,7 \mathrm{c}$ & $32,9 a b$ & 40 & $177,0 \mathrm{c}$ & $213,5 b$ & 45,2 \\
\hline & 7390 & $6,4 a b$ & $2,6 \mathrm{c}$ & $37,8 \mathrm{ab}$ & 41,9 & $192,8 \mathrm{bc}$ & $213,5 b$ & 47,4 \\
\hline & 7602 & $2,0 \mathrm{~d}$ & $4,1 \mathrm{a}$ & $31,2 b$ & 39,2 & $254,3 a b$ & $319,2 \mathrm{a}$ & 44,2 \\
\hline & 8492 & $7,2 \mathrm{a}$ & $3,4 \mathrm{ac}$ & $35,2 a b$ & 49,8 & $240,3 \mathrm{ac}$ & 337,6 a & 42,2 \\
\hline & 8608 & 7,0 a & $3,6 a b$ & $38,9 a$ & 50,3 & 277,6 a & 357,3 a & 43,6 \\
\hline & 8550 & $4,6 \mathrm{bc}$ & $2,7 \mathrm{c}$ & $36,0 \mathrm{ab}$ & 43,4 & $194,5 \mathrm{bc}$ & $232,1 \mathrm{~b}$ & 45,6 \\
\hline
\end{tabular}

fueron ELAD, ELAB, ESAB y \% AD, siendo la introducción 7958 la que presentó mayor densidad estomática por mm lineal en ambas caras de la hoja y por unidad de superficie en la cara abaxial. Para $\% \mathrm{AD}$, al contrario de lo observado en Catamarca, la introducción 7000 fue la que presento mayor proporción de estomas en la epidermis adaxial de la lámina, principalmente por su mayor valor de ESAD en este ambiente (Tabla 5).

En el análisis de correlación entre los valores registrados y las precipitaciones en el sitio de origen, para $T$. crinita se observó que la gran

Tabla 5. Análisis de varianza para los parámetros estomáticos en las introducciones de T. pluriflora en cada ambiente de evaluación. Valores con letras distintas indican diferencias significativas (Tukey, $p<0,05)$.

\begin{tabular}{|c|c|c|c|c|c|c|c|c|}
\hline Ambiente & Accesión & $\begin{array}{c}\mathrm{AL} \\
(\mathrm{mm})\end{array}$ & $\begin{array}{c}\text { DRI } \\
\left(\mathrm{mm}^{1}\right)\end{array}$ & $\begin{array}{c}\text { ELAD } \\
\text { (estomas } / \mathrm{mm} \text { ) }\end{array}$ & $\begin{array}{c}\text { ELAB } \\
\text { (estomas/mm) }\end{array}$ & $\begin{array}{c}\text { ESAD } \\
\left(\text { estomas } / \mathrm{mm}^{2} \text { ) }\right.\end{array}$ & $\begin{array}{c}\text { ESAB } \\
\left(\text { estomas } / \mathrm{mm}^{2}\right)\end{array}$ & $\% A D$ \\
\hline \multirow{6}{*}{ Catamarca } & 7000 & 11,4 & 2,9 & 29,8 & 42,9 & 157 & 246,7 & $0,39 \mathrm{c}$ \\
\hline & 7491 & 9,8 & 3,3 & 31,6 & 36,2 & 189,1 & 238,3 & $0,44 a$ \\
\hline & 7958 & 9,2 & 3,2 & 30,7 & 37,8 & 171,4 & 234,7 & $0,42 a b$ \\
\hline & 7967 & 8,6 & 3,2 & 28,8 & 33,7 & 163,1 & 214,4 & $0,43 a b$ \\
\hline & 8419 & 10 & 2,9 & 29,7 & 37,2 & 153,5 & 214,9 & $0,42 a b$ \\
\hline & 8466 & 11,4 & 3,1 & 29,7 & 38,8 & 167,2 & 238,5 & $0,41 a b$ \\
\hline \multirow{6}{*}{ Esperanza } & 7000 & 10,6 & 3,4 & $33,5 a b$ & $37,8 a b$ & 222,8 & $259,5 a b$ & $0,46 a$ \\
\hline & 7491 & 10,2 & 3,4 & $31,6 a b$ & $34,7 \mathrm{~b}$ & 194,9 & 234,8 b & $0,45 a b$ \\
\hline & 7958 & 10,4 & 3,5 & $35,2 \mathrm{a}$ & $44,2 \mathrm{a}$ & 223,7 & 305,7 a & $0,42 \mathrm{~b}$ \\
\hline & 7967 & 9,8 & 3,6 & $28,9 \mathrm{~b}$ & $36,7 \mathrm{~b}$ & 192,7 & $267,3 a b$ & $0,42 \mathrm{~b}$ \\
\hline & 8419 & 10,2 & 3,6 & $31,7 a b$ & $39,1 a b$ & 208,3 & $278,9 a b$ & $0,43 a b$ \\
\hline & 8466 & 10,5 & 3,5 & $31,9 a b$ & $39,2 a b$ & 202,2 & $270,7 \mathrm{ab}$ & $0,43 a b$ \\
\hline
\end{tabular}


mayoría de las variables presentaron correlación significativa dentro de cada ambiente de evaluación. La variable \%AD presentó correlación negativa para ambas especies, aunque solamente en el ambiente Esperanza para T. crinita, y únicamente en Catamarca para $T$. pluriflora (Tabla 6). Las correlaciones positivas indicaron un mayor ancho de lámina y densidad estomática en aquellos materiales procedentes de sitios con mayores precipitaciones, mientras que se espera una menor proporción de estomas en la superficie adaxial en aquellas introducciones procedentes de ambiente con mayores precipitaciones, ante determinadas condiciones ambientales según la especie.

\section{Discusión}

Este estudio demostró la existencia de patrones de variación respecto de parámetros de densidad y distribución estomática en germoplasma nativo de Trichloris, presentando diferente relación con el régimen hídrico según el nivel de análisis. En el ACP resultó evidente que el efecto ambiental del sitio de evaluación tiene un importante efecto sobre la gran mayoría de las variables analizadas. Dejando de lado las diferencias entre especies, las variaciones para ancho de la lámina no evidenciaron asociación con el ambiente de evaluación, sugiriendo para este carácter la ausencia de plasticidad fenotípica significativa en respuesta a las condiciones de crecimiento. En el mismo sentido, Greco \& Cavagnaro (2003), reportaron que las diferencias de morfología foliar entre diferentes genotipos de $T$. crinita no fueron alteradas al variar las condiciones hídricas en el ambiente de evaluación. Estas observaciones, sumadas a la presencia de correlación positiva entre el ancho de lámina y las precipitaciones anuales del sitio de origen, sugieren que este carácter estaría marcadamente condicionado por el ambiente de procedencia. En general, la disminución de la superficie foliar constituye una estrategia de adaptación al estrés hídrico muy extendida en el reino vegetal. Las hojas estrechas resultan más eficientes en el intercambio de calor y de esta manera favorecen el balance de temperatura en la planta (Redman, 1985). Las especies que se encuentran con mayor frecuencia en ambientes xerófitos normalmente presentan hojas pequeñas y/o enrolladas (Parkhust, 1978), por lo que esas adaptaciones son consideradas como

\begin{tabular}{|c|c|c|c|c|c|}
\hline \multirow{2}{*}{ Especie } & \multirow{2}{*}{ Variables } & \multicolumn{4}{|c|}{ Ambiente } \\
\hline & & \multicolumn{2}{|c|}{ Catamarca } & \multicolumn{2}{|c|}{ Esperanza } \\
\hline \multirow{7}{*}{ T. crinita } & AL & 0,55 & $* *$ & 0,43 & * \\
\hline & RI/AL & 0,42 & * & 0,46 & * \\
\hline & ELAB & 0,46 & * & 0,52 & ** \\
\hline & ELAD & 0,17 & ns & 0,21 & ns \\
\hline & ESAB & 0,53 & $* *$ & 0,76 & ** \\
\hline & ESAD & 0,31 & * & 0,62 & ** \\
\hline & $\% A D$ & $-0,03$ & ns & $-0,39$ & * \\
\hline \multirow{7}{*}{ T. pluriflora } & AL & 0,34 & ns & 0,06 & ns \\
\hline & RI/AL & $-0,26$ & ns & 0,08 & ns \\
\hline & ELAB & 0,17 & ns & 0,14 & ns \\
\hline & ELAD & $-0,12$ & ns & $-0,03$ & ns \\
\hline & ESAB & $-0,04$ & ns & 0,18 & ns \\
\hline & ESAD & $-0,25$ & ns & 0,05 & ns \\
\hline & $\% A D$ & $-0,43$ & * & $-0,17$ & ns \\
\hline
\end{tabular}

estrategias adaptativas que adoptan para minimizar el proceso de transpiración (Fahn \& Cutler, 1992). Por ello, sería recomendable analizar a futuro en Trichloris si la asociación del ancho foliar con las precipitaciones anuales en origen resulta un indicador útil de la tolerancia a estrés hídrico, mediante análisis de parámetros fisiológicos, como la eficiencia fotosintética o el índice de área foliar.

La densidad de regiones intercostales presentó una singular variación y plasticidad, registrando diferencias significativas en todos los niveles de análisis. No existen antecedentes de estudios de variaciones para este carácter ante diferentes condiciones ambientales, aunque para el género Trichloris la región intercostal ha sido descripta en función de particularidades anatómicas (Gil et al., 1992; Pérez Cuadra \& Cambi, 2010). Si bien para ambas especies los registros de DRI fueron mayores en ambiente húmedo, para $T$. pluriflora se observaron los mayores valores tanto en general como para cada ambiente por separado. Resulta evidente que los valores de DRI y sus variaciones no están asociados al ancho de la lámina, llegando 


\section{A. Trod et al. - Distribución y densidad estomática en Trichloris}

incluso a variar en forma inversa para la introducción 7602 de $T$. crinita, la cual además constituye un ecotipo morfológicamente muy contrastante al resto de los materiales analizados de la especie (Trod, M.A., com. pers). Para este caso en particular, una posible explicación podría asociarse a una modificación en la intercepción de la radiación ante ausencia de estrés hídrico como mecanismo de compensación, requiriéndose estudios específicos a futuro para verificarlo.

Tanto T. crinita como T. pluriflora presentan estomas distribuidos en ambas superficies de la lámina, por lo que pueden ser consideradas como especies anfi-estomáticas (Perez Cuadra \& Cambi, 2014). En varios estudios se ha demostrado que la distribución y densidad de estomas sobre la superficie foliar están relacionadas con la adaptación al estrés hídrico, la temperatura y exposición foliar (Parkust, 1978; Smith et al. 1997, 1998). Además, alteraciones en la densidad estomática mediadas por condiciones experimentales han demostrado que ambas superficies foliares varían en forma independiente (Muir et al., 2015). En este estudio se observaron diferencias entre especies de Trichloris para las variables de densidad estomática lineal, tanto en la superficie adaxial (solo en ambiente Esperanza) como en la abaxial (en general y en Esperanza), siendo siempre mayor la densidad en $T$. crinita. Por otro lado, al particionar por especie, en ambas se observaron diferencias significativas entre ambientes solamente para la superficie adaxial. Nuestras observaciones sugieren que existe mayor plasticidad fenotípica para la densidad estomática lineal en la superficie adaxial para ambas especies, siendo dicha plasticidad de mayor magnitud en $T$. crinita.

La información discutida hasta aquí indica que en Trichloris la distribución de estomas en la lámina foliar responde a diferentes patrones según la especie: en $T$. crinita se encuentra una menor densidad de regiones intercostales pero con alta densidad estomática lineal, mientras que en $T$. pluriflora se observó lo opuesto, mayor densidad de regiones intercostales pero con baja densidad estomática lineal. De este modo, ambos parámetros se compensan mutuamente. Esto explicaría el por qué, al analizar la densidad estomática por unidad de superficie en ambas caras de la hoja y la proporción de estomas en la superficie adaxial, no se observaron diferencias significativas entre especies, sino que difirieron entre ambientes al particionar por especie, presentando valores más altos en el ambiente húmedo. Diversos estudios indican que, en especies o ecotipos no adaptados a ambientes secos, la densidad estomática se correlaciona negativamente al someterlos a condiciones de estrés hídrico (Ristic \& Cass, 1991; Bastos et al., 1993; Fraser et al., 2009), mientras que, por el contrario, para las especies de ambiente árido la reducción en la densidad estomática representa una adaptación que aumenta la eficiencia del uso del agua (Xu \& Zhou, 2008; De Micco \& Aronne, 2012; Ashraf \& Harris, 2013). Dado que ambas especies de Trichloris habitan ambientes con problemas de estrés hídrico, la densidad estomática podría representar un indicador de la eficiencia en el uso del agua para los materiales bajo estudio. Las especies anfiestomáticas presentan estomas en ambas superficies foliares, predominando aquellas con mayor densidad estomática en la superficie abaxial (Bucher et al., 2017). En especies propias de ambientes áridos y semiáridos o de alta irradiancia, la densidad de estomas suele ser similar en ambas superficies foliares respecto de especies que vegetan en ambientes húmedos o sombreados (James \& Bell, 2000; Volenikova \& Ticha, 2001; Tari 2003; Bucher et al., 2017). En coincidencia con esos estudios, los resultados aquí presentados indican que en ambas especies de Trichloris también es menor la proporción de estomas en la superficie adaxial, diferencia que se acentúa ante condiciones de mayor estrés. La distribución de los estomas es un factor determinante de la efectividad del plegamiento foliar para reducir la pérdida de agua por transpiración, lo que define la estrategia ecológica de la especie (Redmann, 1985). Al igual que lo observado para la densidad estomática lineal, las variables de distribución relativa y densidad estomática por unidad de superficie presentaron mayor la plasticidad para la superficie adaxial y, en particular, en $T$. crinita. Dado que $T$. crinita manifiesta plegamiento foliar ante condiciones de estrés hídrico (Greco \& Cavagnaro, 2003), las variaciones de densidad y distribución estomática entre materiales y su plasticidad estarían indicando un mayor potencial adaptativo para esta especie, en relación a $T$. pluriflora. Existe también la posibilidad que esta tendencia general del análisis responda a la existencia de mayor variabilidad dentro de la colección de $T$. crinita debido a un muestreo más extenso del área de distribución de la especie y abarcando diferentes regiones 


\section{Bol. Soc. Argent. Bot. 53 (4) 2018}

fitogeográficas, a diferencia de T. pluriflora cuyas introducciones proceden de ambientes menos diferenciados entre sí.

En la comparación entre introducciones de cada especie, para T. crinita se observó un mayor nivel de variación intraespecífico respecto de $T$. pluriflora, en coincidencia con las observaciones antes planteadas. Otros estudios comparativos realizados en $T$. crinita indican que los materiales originarios de sitios con mayor déficit hídrico son menos afectados por condiciones de evaluación en sequía, mostrando mayor producción de biomasa, potencial hídrico de la hoja y aplazamiento de los signos visuales de estrés por sequía (Kozub et al., 2017). Nuestros resultados indican que el ambiente Catamarca permitió visualizar mejor las diferencias entre introducciones y sugieren que los materiales procedentes de ambiente más seco son capaces de variar en mayor medida su densidad estomática, lo que podría trasuntar en una mayor eficiencia en el uso del agua. Aunque estudios en otras especies indican que la densidad estomática no presentó correlación con el área foliar (Fraser et al., 2009), se requieren estudios para analizar en Trichloris la asociación entre la densidad estomática y la producción de biomasa en ambiente restrictivo.

Para T. pluriflora, las diferencias entre introducciones se observaron en Esperanza, por lo que el ambiente húmedo resultó de mayor relevancia para expresar las variaciones del germoplasma analizado. Se destacó la introducción 7000, que registró elevados niveles de plasticidad entre ambientes de evaluación para la proporción relativa de estomas entre las superficies foliares. Teniendo en cuenta también lo observado para $T$. crinita, el ambiente de evaluación resultó de suma importancia para analizar las diferencias entre los materiales que integran la colección, obteniéndose mejores resultados en sitios que mejor representen al lugar de origen de las introducciones.

Existen numerosos estudios donde se ha determinado la existencia de asociación entre la densidad estomática con factores ambientales del lugar de procedencia. En el caso de las precipitaciones en el sitio de origen, la reducción en la densidad estomática constituiría una adaptación que aumenta la eficiencia del uso del agua (Xu \& Zhou, 2008; De Micco \& Aronne, 2012; Ashraf \& Harris, 2013; Cach-Pérez et al., 2016). Este aspecto ha llevado a que la densidad estomática sea considerada un criterio de selección, la manipulación genética de la densidad estomática sea un objetivo de mejoramiento actual (Hepworth et al., 2015; Hughes et al., 2017). En este estudio, la correlación entre la mayoría de las variables analizadas y las precipitaciones anuales en el lugar de origen fue significativa para ambos ambientes de evaluación para $T$. crinita. Esto sugiere que, para esa especie, las precipitaciones tienen gran influencia sobre variabilidad genética para la densidad estomática y las dimensiones foliares, como así también sobre su plasticidad. Para T. pluriflora, por el contrario, las precipitaciones en el ambiente de procedencia no explicaron el comportamiento de las introducciones analizadas. La correlación negativa en la proporción de estomas en cada superficie foliar respecto de las precipitaciones en origen solo fue relevante en uno de los ambientes de evaluación, diferente para cada especie. El hecho de que en ambiente húmedo presenten mayor proporción de estomas en la superficie adaxial aquellas introducciones de $T$. crinita procedentes de ambiente más seco, indica que dichos materiales presentan mayor plasticidad para el carácter. En forma análoga para T. pluriflora, las introducciones procedentes de ambiente húmedo presentarían mayor plasticidad en cuanto a la proporción relativa de la distribución estomática. Estos aspectos podrían ser de relevancia para programas de mejora genética en la especie.

En el presente trabajo se ha analizado la variación en la densidad estomática en respuesta a diferentes condiciones hídricas para dos especies que, actualmente en base a estudios de filogenia molecular, se incluyen dentro del género Leptochloa. En el mismo sentido, estudios realizados para Leptochloa reportan la ocurrencia de reducción en el número de estomas en condiciones de crecimiento en salinidad (Ola et al., 2012). Por ello, se considera importante realizar a futuro estudios en especies nativas del género, para analizar posibles variaciones de su anatomía foliar frente condiciones ambientales adversas, identificar los mecanismos involucrados en el proceso de adaptación a tales condiciones y determinar su importancia como recursos para ambientes con limitantes productivas y como fuente de variabilidad para la tolerancia estreses abióticos.

Como conclusión, la diversidad analizada ha permitido identificar variables morfológicas de importancia para caracterizar la colección, fundamentalmente en base a su posible relación con estrategias de adaptación a un ambiente restrictivo 


\section{A. Trod et al. - Distribución y densidad estomática en Trichloris}

y la plasticidad fenotípica manifestada. El ambiente de evaluación resultó un elemento importante a la hora de potenciar la expresión de las diferencias entre especies y entre materiales de cada especie. Las diferencias entre las introducciones permitieron identificar a aquellos fenotipos contrastantes y remarcar la importancia de factores ambientales como las precipitaciones en el lugar de origen para la programación de estrategias de colecta y conservación del recurso fitogenético.

\section{Agradecimientos}

Al Ing. Agr. Emiliano Quiroga por facilitar las instalaciones del INTA Catamarca para la implantación del ensayo y por su colaboración en el mantenimiento del mismo, y a los revisores anónimos por sus contribuciones y sugerencias. Este estudio fue financiado por CAI+D $2011 \mathrm{UNL}$ $\mathrm{N}^{\circ}$ 50120110100234LI.

\section{Bibliografía}

ASHRAF, M. \& P. J. C. HARRIS. 2013. Photosyntesis under stressful environments: An overview. Photosyntetica 51: 163-190.

BARBOZA, S. B. S. C., D. G. RIBEIRO, J. B. TEIXEIRA, T. A. PORTES \& L. A. C. SOUZA. 2006. Anatomía foliar de plantas micropropagadas de abacaxi. Pesq. Agropec. Bras. 41: 185-194.

BARUCH, Z. \& D. S. FERNANDEZ. 1993. Water relations of native and introduced $\mathrm{C} 4$ grasses in a neotropical savanna. Oecologia 96: 179-185.

BASTOS, E.O., M. PERAZZOLO \& J. M. R. BATISTA. 1993. Variação do número de estômatos e micropelos em Paspalum vaginatum em relação às condições abióticas numa marisma do estuário da Lagoa dos Patos, RS, Brasil. Acta Bot. Bras. 6: 105-117.

BUCHER, S. F., K. AUERSWALD, C. GRÜN-WENZEL, S. I. HIGGINS, J. C. JORGE \& C. RÖMERMANN. 2017. Stomatal traits relate to habitat preferences of herbaceous species in a temperate climate. Flora 229: 107-115.

CACH-PÉREZ, M. J., J. L. ANDRADE, W. CETZALIX \& C. REYES-GARCÍA. 2016. Environmental influence on the inter- and intraspecific variation in the density and morphology of stomata and trichomes of epiphytic bromeliads of the Yucatan Peninsula. Bot. J. Linn. Soc. 181: 441-458.

CARBUTT, C., W. HENWOOD \& L. GILFEDDER. 2017. Global plight of native temperate grasslands: going, going, gone? Biodivers. Conserv. 26: 2911-2932.
DE MICCO, V. \& G. ARONNE. 2012. Morphoanatomical traits for plant adaptation to drought: from morphological to molecular features. In: AROCA R. (ed.), Plant Responses to Drought Stress, pp. 37-61. Springer, Berlin, Heidelberg.

DI RIENZO, J. A., F. CASANOVES, M. G. BALZARINI, L. GONZALEZ, M. TABLADA \& C. W. ROBLEDO. 2018. InfoStat, versión 2018. Grupo InfoStat, FCA, Universidad Nacional de Córdoba, Argentina. URL http://www.infostat.com.ar

FAHN, A. \& D. F. CUTLER. 1992. Encyclopedia of plant anatomy. Vol XIII: Xerophytes. Berlín, Gebruder Borntraeger.

FAO. 2008. Informe nacional sobre el estado de los recursos fitogenéticos para la agricultura y la alimentación. Argentina. [online]. Disponible en: http://www.fao.org/ docrep/013/i1500e/Argentina.pdf. [Acceso: 17/05/18].

FRASER, L. H., A. GREENALL, C. CARLYLE, R. TURKINGTON \& C. R. FRIEDMAN. 2009. Adaptive phenotypic plasticity of Pseudoroegneria spicata: response of stomatal density, leaf area and biomass to changes in water supply and increased temperature. Ann. Bot.103: 769-775.

GIL, P., S. M. PONS \& G. M. RUIZ. 1992. Identificación en base a características epidérmicas de especies forrajeras nativas del bosque chaqueño occidental de la provincia de Córdoba. I. Poaceas. Agriscientia 9: 31-43.

GONZALEZ MEDRANO, F. G. 2012. Las zonas áridas y semiáridas de México y su vegetación. Instituto Nacional de Ecología.

GRECO, S. A. \& J. B. CAVAGNARO. 2003. Effect of drought in biomass production and allocation in three varieties of Trichloris crinita (Poaceae) a forage grass from the arid Monte region of Argentina. Plant Ecol. 164: $125-135$.

GUTIÉRREZ, H. F., G. A. RICHARD, M. C. CERINO \& J. F. PENSIERO. 2016. Sistema reproductivo de Trichloris (Poaceae, Chloridoideae, Chlorideae). Bol. Soc. Argent. Bot. 51: 111-122.

HENWOOD, W. 2010. Toward a strategy for the conservation and protection of the world's temperate grasslands. Great Plains Res. 20: 121-134.

HEPWORTH, C., T. DOHENY-ADAMS, L. HUNT, D. D. CAMERON \& J. E. GRAY. 2015. Manipulating stomatal density enhances drought tolerance without deleterious effect on nutrient uptake. New Phytol. 208: 336-341.

HONG Z., K. LAKKINENI, Z. ZHANG \& D. P. S. VERMA. 2000. Removal of feedback inhibition of 1-pyrroline-5-carboxylate synthetase results in increased proline accumulation andprotection of plants from osmotic stress. Plant Physiol. 122: 1129-1136.

HUGHES, J., C. HEPWORTH, C. DUTTON, J. A. DUNN, L. HUNT, J. STEPHENS, R. WAUGH, D. CAMERON \& J. E. GRAY. 2017. Reducing stomatal density in barley improves drought tolerance without impacting on yield. Plant physiol. 174: 776-787. 
JAMES, A. S \& D. T. BELL. 2000. Influence of light availability on leaf structure and growth of two Eucalyptus globulus ssp. globulus provenances. Tree Physiology 20: 1007-1018.

KAISER, W. M. 1987. Effects of water deficit on photosynctetic capacity. Physiol. Plant. 71: 142-149.

KOZUB, P. C., K. BARBOZA, F. GALDEANO, C. L. QUARIN, J. B. CAVAGNARO \& P. F. CAVAGNARO. 2017. Reproductive biology of the native forage grass Trichloris crinita (Poaceae, Chloridoideae). Plant Biol. 19: 444-453.

LUDLOW, M. M. \& T. T. NG. 1976. Effect of water deficit on carbon dioxide exchange and leaf elongation rate of Panicum maximun var. trichoglume. Functional Plant Biol. 3: 401-413.

MARINONI, L., A. BORTOLUZZI, M. PARRAQUIJANO, J. M. ZABALA \& J. F. PENSIERO. 2015. Evaluation and improvement of the ecogeographical representativeness of a collection of the genus Trichloris in Argentina. Genet. Resour. Crop Evol. 62: 593-604.

MUIR, C. D. 2015. Making pore choices repeated regime shifts in stomatal ratio. Proc. $R$. Soc. B. 282: 20151498.

OLA, H. A. E., E. F. REHAM, S. S. EISA \& S. A. HABIB. 2012. Morpho-anatomical changes in salt stressed kallar grass (Leptochloa fusca L. Kunth). Res. J. Agric. Biol. Sci. 8: 158-166.

PARKHUST, D. 1978. The adaptative significance of stomatal occurrence on one or both surfaces of leaves. J. Ecol. 66: 367-383.

PASSERA, C., J. B. CAVAGNARO, J. LEMES \& L. ALLEGRETTI. 1997. Gramíneas nativas de zonas áridas, banco de germoplasma y selección de ecotipos en el Monte, Argentina. Actae Etnobotánica 92: 181-189.

PEREZ CUADRA, V. \& V. CAMBI. 2010. Anatomía vegetativa comparativa entre Chloridoideae (Poaceae) halófitas de importancia forrajera. Phyton 79: 69-76.

PEREZ CUADRA, V. \& V. CAMBI. 2014. Ocurrencia de caracteres anatómicos funcionales foliares y caulinares en 35 especies Xero-halófilas. Bol. Soc. Argent. Bot. 49: 347-359.

PETERSON, P. M., ROMASCHENKO, K., SNOW, N., \& G. JOHNSON. 2012. A molecular phylogeny and classification of Leptochloa (Poaceae: Chloridoideae: Chlorideae) sensu lato and related genera. Ann Bot. 109: 1317-1330.
REDMANN, R.E. 1985. Adaptation on grasses to water stress-leaf rolling and stomata distribution. Ann. Missouri Bot. Gard. 72: 833-842.

RISTIC, Z. \& D. D CASS. 1991. Leaf anatomy of Zea mays L. in response to water shortage and high temperature: a comparison of drought-resistant and drought-sensitive lines. Bot. Gaz. 152: 173-185.

SHARMA, G. K. 1972. Environmental modifications of leaf epidermis and morphological features in Verbena canadensis. Sout. Nat. 221-228.

SMITH, W. K., T. C. VOGELMANN, E. H. DE LUCIA, D. T. BELL \& K. A. SHEPHERD. 1997. Leaf form and photosynthesis. Bioscience 47: 785-793.

SMITH, W. K., D. T. BELL \& K. A. SHEPHERD. 1998. Associations between leaf structure, orientation and sunlight exposure in five Western Australian communities. Am. J. Bot. 85: 56-63.

TARI, I. 2003. Abaxial and adaxial stomatal density, stomatal conductances and water status of bean primary leaves as affected by paclobutrazol. Biol. Plant. 47: 215-220.

VILlAGRA, P. E., C. GIORDANO, J. A. ALVAREZ, J. B. CAVAGNARO, A. GUEVARA, C. SARTOR, C. PASSERA \& S. GRECO. 2011. Ser planta en el desierto: estrategias de uso de agua y resistencia al estrés hídrico en el Monte Central de Argentina. Ecología austral 21: 29-42.

VOLENÍKOVÁ, M. \& I. TICHÁ. 2001. Insertion profiles in stomatal density and sizes in Nicotiniana tabacum L. plantles. Biol. Plant. 44: 161-165.

XU, Z. \& G. ZHOU. 2008. Responses of leaf stomatal density to water status and its relationships with photosynthesis in a grass. J. Exp. Bot. 59: $3317-$ 3325 .

ZARLAVSKY, G. E. 2014. Histología Vegetal: técnicas simples y complejas. Sociedad Argentina de Botánica, Buenos Aires.

ZULOAGA, F. O., E. G. NICORA, Z. E. RÚGOLO DE AGRASAR, O. MORRONE, J. F. PENSIERO \& A. M. CIALDELLA. 1994. Catálogo de la familia Poaceae en la República Argentina. Missouri Botanical Garden. Monog. Syst. Bot. 47: 1-178.

Recibido el 18 de junio de 2018, aceptado el 10 de octubre de 2018. Editora: Ana M. Gonzalez. 